\title{
Relaciones entre el autoconcepto y el perfil psicológico deportivo en triatletas
}

\section{Relationship between self-concept and psychological sport profile in triathletes}

\section{As relaçóes entre auto-conceito e perfil psicológico atletas de esportes}

\author{
Rafael López Cazorla², Antonio Hernández Mendo², Rafael Enrique Reigal Garrido³ y Verónica Morales Sánchez² \\ ${ }^{1}$ Facultad de Psicología, Universidad de Málaga (España), ${ }^{2}$ Departamento de Psicología Social, Facultad de Psicología, Universidad de Málaga (España) y ${ }^{3}$ \\ Grupo Investigación CTS-642, Universidad de Granada (España).
}

Resumen: El propósito de este trabajo fue examinar las relaciones entre el autoconcepto multidimensional y el perfil psicológico en un grupo de triatletas. Participaron en el estudio 88 triatletas con edades entre 20 y 55 ańos $(M=36.06$; $\mathrm{DT}=7.75)$. Se utilizó el Cuestionario Autoconcepto Forma 5 (AF5) para evaluar el autoconcepto y el Inventario Psicológico de Ejecución Deportiva (IPED) para analizar el perfil psicológico. Los análisis de correlación efectuados pusieron de manifiesto relaciones significativas entre los constructos estudiados, siendo el autoconcepto emocional el que tuvo una mayor correlación con los diferentes factores del perfil psicológico. Además, la autoconfianza, el control de afrontamiento negativo y el control actitudinal fueron las dimensiones del IPED que obtuvieron un mejor valor de predicción a partir de los diferentes factores del autoconcepto. Los resultados hallados pusieron de manifiesto la importancia del autoconcepto en la configuración de las diversas habilidades psicológicas implicadas en el rendimiento del deportista.

Palabras Clave: perfil psicológico; autoconcepto; triatletas.

Abstract: The purpose of this study was to examine the relationship between self-concept and psychological profile on a sample of triathletes. Participated in the study 88 athletes aged between 20 and 55 years $(M=36.06$, $\mathrm{SD}=7.75)$. The "Autoconcepto Forma 5" questionnaire (AF5) was used to evaluate the multidimensional self-concept and the Inventory of Sports Performance (IPED) was used to analyze the psychological profile. The correlation analysis performed showed significant relationships between the constructs studied and highlighting that the emotional self-concept was the variable which had the strongest association with IPED factors. In addition, self-confidence, negative coping control and attitudinal control were the dimensions of the IPED who obtained a better predictive value from the different factors of self-concept. The results found showed the importance of self-concept in the configuration of the psychological skills involved in the athlete's performance.

Keywords: psychological profile; self-concept; triathletes.

Resumo: $\mathrm{O}$ objetivo deste estudo foi examinar a relaçáo entre o auto-conceito multidimensional e perfil psicológico de um grupo de triatletas. Participaram do estudo 88 atletas com idade entre 20 e 55 anos $(M=36,06$, $\mathrm{DP}=7,75)$. Para avaliar o auto-conceito foi usado o Questionário do Auto-conceito Forma 5 (AF5) e para analisar o perfil psicológico foi utilizdo o Inventario de Execução Deportiva (IPED). A análise de correlação realizados mostram relaçóes significativas entre os construtos estudados, sendo o eu emocional que teve maior correlaçáo com os diferentes fatores do perfil psicológico. Além disso, a auto-confiança, controle de enfrentamento negativo e controle atitudinais foram dimensōes do IPED que obteve uma previsão melhor valor dos diferentes fatores de auto-conceito. Os resultados obtidos mostraram a importância da auto-conceito na configuraçáo das várias capacidades psicológicas envolvidas no desempenho do atleta.

Palavras-chave: perfil psicológico; auto-conceito; triatletas.

\section{Introducción}

Conocer el perfil psicológico del deportista es importante para mejorar los procesos de entrenamiento y las expectativas de éxito en la competición (López-Gullón et al., 2012). De hecho, son diversas las habilidades psicológicas que se consideran fundamentales para predecir el rendimiento en el deporte tales como la autoconfianza, los niveles motivacionales, el control emocional o las estrategias de afrontamiento (Berengüí et al., 2012). Entre otros aspectos, el conocimiento de estas variables y la capacidad de intervenir sobre ellas puede controlar procesos como los estados de ansiedad o la persistencia para alcanzar un objetivo (Arruza, González, Palacios, Arribas y Cecchini, 2012; Massuça, Fragoso y Teles, 2014;

Dirección para correspondencia [Correspondence address]: Rafael López Cazorla. Facultad de Psicología, Universidad de Málaga (Espańa) Campus de Teatinos, 29071, Málaga (España). Email: rlcazorla69@ gmail.com
Parry, Chinnasamy, Papadopoulou, Noakes y Micklewright, 2011), lo cual se está aplicando en el ámbito de la psicología del deporte y constituye un interesante campo de investigación (Garcia-Mas et al., 2011; Urra, 2004).

En este sentido, Fernandes, Bombas, Lázaro y Vasconcelos-Raposo (2007) analizaron el perfil psicológico en una muestra de adolescentes que participaban en el deporte de vela hallando que la capacidad de visualización y la motivación predecían significativamente el rendimiento en esta disciplina. En otro trabajo, López-Gullón et al. (2011) pusieron de manifiesto que las variables autoconfianza y control actitudinal permitían diferenciar a luchadores de élite y amateur en las disciplinas Libre Olímpica y Grecorromana. Asimismo, Heazlewood y Burke (2011) observaron en un grupo de triatletas que el rendimiento en una prueba de triatlón ironman se podía explicar, entre otros factores, a partir de las autoevaluaciones de percepción eficacia de los deportistas. Asimismo, 
Nicholls y Polman (2007) efectuaron una revisión de las estrategias de afrontamiento psicológicas, poniendo de relieve su implicación en el comportamiento de los deportistas en relación a la competición.

Uno de los instrumentos disponibles para efectuar este tipo de análisis es el Inventario Psicológico de Ejecución Deportiva (IPED) (Hernández-Mendo, 2006; Hernández-Mendo, Morales-Sánchez y Peñalver, 2014), adaptación al castellano del Psychological Performance Inventory (PPI) de Loehr (1986, 1990). Este instrumento recoge una amplia información sobre diversas habilidades psicológicas relacionadas con la competición deportiva y ha sido utilizado para analizar estos constructos en diversas investigaciones (Berengüí et al., 2012; Gucciardi, 2012). Como ejemplo, López-Gullón et al. (2012) encontraron, en la lucha olímpica, que los deportistas de élite tenían puntuaciones significativamente mejores en autoconfianza y control actitudinal. Asimismo, Hernández-Mendo (2006) y Hernández-Mendo, Morales-Sánchez y Peñalver (2014) recogieron las puntuaciones obtenidas en las diferentes escalas del IPED por participantes en diversas modalidades deportivas.

El perfil psicológico del deportista puede estar condicionado por otros elementos, como los rasgos de personalidad (Allen, Frings y Hunter, 2012; Laborde, Dosseville y Kinrade, 2014) o las propias evaluaciones que efectúan sobre sus capacidades (Jackson, Thomas, Marsh y Smethurst, 2001). En el conjunto de juicios que las personas realizan sobre ellas, el autoconcepto es un constructo que se refiere al concepto que el ser humano tiene sobre sí mismo, el cual ha sido asociado a un mejor funcionamiento en diferentes ámbitos y se ha considerado un determinante de éxito deportivo (Esnaola, Goñi y Madariaga, 2008; Guillén y Ramírez, 2011; Slutzky y Simpkins, 2009). Actualmente, el modelo de autoconcepto más extendido hace referencia a una realidad de naturaleza multidimensional en la que se puede diferenciar su vertiente física, emocional, familiar, académica o social (Esnaola, Rodríguez y Goñi, 2011; Shavelson, Hubner y Stanton, 1976).

Son numerosas las evidencias que han puesto de relieve los vínculos entre el autoconcepto y algunas habilidades psicológicas en contextos no deportivos. Como ejemplo, existen investigaciones que han observado que diversas estrategias de afrontamiento estaban relacionadas con el autoconcepto en niños y adolescentes (Sung, 2011; Zhou, Wu y Lin, 2012). Asimismo, otros trabajos han encontrado relaciones entre algunas dimensiones del autoconcepto y la motivación en el ámbito educativo (Eyal y Roth, 2011; Faye y Sharpe, 2008). En el contexto de la práctica física y deportiva, aunque no existe un conjunto amplio de estudios que hayan analizado las relaciones entre estos constructos, sobre todo en el ámbito del rendimiento deportivo, sí se han hallado algunos datos que han manifestado la relación entre el autoconcepto y as- pectos como el nivel de motivación o la confianza para realizar este tipo de tareas (Martín-Albo, Núñez, Domínguez, León y Tomás, 2012; McDonough y Crocker, 2005; Wang, Liu, Lochbaum y Stevenson, 2009). Además, la asociación entre el autoconcepto y factores como el estado de flow experimentado en el deporte, la perseverancia en el esfuerzo desarrollado o la motivación con la que se practica (Amesberger, Finkenzeller, Würth y Müller, 2011; Jackson et al., 2001; Luszczynska y Abraham, 2012), sugiere la influencia que el concepto de sí mismo puede tener sobre las habilidades psicológicas implicadas en la ejecución deportiva.

Uno de los deportes más exigentes que existen en la actualidad es el triatlón, compuesto por tres disciplinas, natación, ciclismo y carrera a pie (Cejuela, Pérez, Villa, Cortell y Rodríguez, 2007). Dada su dureza, este deporte requiere un profundo análisis de las exigencias de las diferentes pruebas, así como de la evaluación de las características de los participantes para ajustar los procesos de entrenamiento y mejorar las prestaciones en la competición. Por ello, son cada vez más numerosas las investigaciones que han intentado obtener datos que contribuyan a comprender mejor su desarrollo y la capacidad del triatleta para adaptarse física y psicológicamente a él (Landers et al., 2013; Lepers, Knechtle y Stapley, 2013; Marongiu et al., 2013; Taylor y Smith, 2013). Sin embargo, aunque existen diversos trabajos que han profundizado en los perfiles psicológicos del triatleta (Jaenes, Peńaloza, Navarrete y Bohórquez, 2012) es un ámbito de estudio relativamente poco explorado, que requiere incrementar las evidencias científicas para su mejor comprensión.

No se han encontrado estudios que analicen la relación entre el autoconcepto multidimensional y el perfil psicológico en triatletas. Por ello, y con el objetivo de comprender mejor la configuración de las habilidades psicológicas en este contexto, el presente trabajo trata de analizar las relaciones entre el autoconcepto multidimensional y diferentes habilidades psicológicas en una muestra de triatletas, así como la posibilidad de predecir a partir de las puntuaciones en autoconcepto los valores del perfil psicológico de los participantes en el estudio.

\section{Método}

\section{Participantes}

Participaron en la investigación 88 triatletas, 76 hombres y 12 mujeres, con edades entre 20 y 55 ańos $(M=36.06$; DT= 7.75). La muestra seleccionada estaba constituida por triatletas que participaban en pruebas de nivel nacional. Se excluyeron del estudio aquellos participantes cuyo deporte principal no era el triatlón, aunque compitiesen ocasionalmente en alguna prueba. 


\section{Instrumentos y material}

a) Cuestionario Autoconcepto Forma 5 (AF5 - García y Musitu, 2001). Este cuestionario, formado por 30 ítems, evalúa el autoconcepto multidimensional y está formado por 5 factores: autoconcepto académico/profesional (e.g., Hago bien los trabajos escolares/profesionales), social (e.g., Hago fácilmente amigos), emocional (e.g., Tengo miedo de algunas cosas), familiar (e.g., Soy muy criticado en casa) y físico (e.g., Me cuido físicamente). Se contesta mediante una escala con puntuaciones entre 1 y 99, en el que 1 significa estar en total desacuerdo y 99 un acuerdo máximo con lo dictado en cada cuestión. Posteriormente esta escala se traslada a una más reducida con puntuaciones entre 1 y 10 para su análisis e interpretación. Los análisis de consistencia interna de la escala ofrecieron para este trabajo un Alfa de Cronbach de ".78" para la escala total y valores similares para cada factor: Académico/profesional =".81"; social = “.88”; emocional = “.76”; familiar = “.83”; físico $=$ ". $.66 "$.

b) Inventario Psicológico de Ejecución Deportiva (IPED; Hernández-Mendo, 2006). Este cuestionario es la adaptación en castellano del Psychological Performance Inventory (PPI) de Loehr $(1986,1990)$ y es empleado para valorar diferentes habilidades del perfil psicológico competitivo del deportista. Constituido por 42 ítems y siete factores: (1) autoconfianza (e.g., Me veo más como un perdedor que como un ganador durante las competiciones), (2) control de afrontamiento negativo (e.g., Me enfado y frustro durante la competición), (3) control atencional (e.g., Llego a distraerme y perder mi concentración durante la competición), (4) control visuimaginativo (e.g., Antes de la competición, me imagino a mi mismo ejecutando mis acciones y rindiendo perfectamente), (5) nivel motivacional (e.g., Estoy muy motivado para dar lo mejor de mí en la competición), (6) control de afrontamiento positivo (e.g., Puedo mantener emociones positivas durante la competición) y (7) control actitudinal (e.g., Durante la competición pienso positivamente). A este cuestionario se responde mediante un continuo con cinco opciones de respuesta de 1 (casi nunca) a 5 (casi siempre). Los análisis de consistencia interna de la escala ofrecieron para este trabajo un Alfa de Cronbach de ".91" para la escala total y valores menores para cada factor (Autoconfianza = ".68"; Control de afrontamiento negativo = ".66"; Control atencional = ".57"; Control visu-imaginativo = “.81”; Nivel motivacional = “.68”; Control de afrontamiento positivo = ".67"; Control actitudinal $=$ “.70”).

\section{Procedimiento}

La captación de la muestra se realizó por dos vías: (1) se contactó con dos federaciones autonómicas, la Federación Andaluza y Valenciana de Triatlón, elaborándose un escrito a estas federaciones donde se explicaba la finalidad de la investigación, pidiéndoles la colaboración para difundir el objetivo investigador a sus triatletas federados a través de mailing y páginas sociales; (2), de forma paralela a la anterior, se realizaron unas octavillas que se difundieron en diversas competiciones de triatlón de la Comunidad Andaluza. Tras la aceptación de los deportistas para participar en el estudio se obtuvo el consentimiento informado. Además, durante todo el proceso de investigación se respetaron los principios establecidos en la declaración de Helsinki (2000).

Los deportistas cumplimentaron los cuestionarios y lo enviaron de forma online a través de la plataforma de evaluación psicosocial MENPAS (González-Ruiz, Hernández-Mendo y Pastrana Brincones, 2010). Los participantes tenían información de contacto de los responsables del estudio para que pudieran resolver cualquier duda. La recepción de los cuestionarios se efectuó entre los meses de marzo y mayo del ańo 2013. Aproximadamente, cada participante rellenó los dos cuestionarios en un tiempo de 20 minutos.

Se ha empleado un diseño de investigación predictivo transversal (Ato, López y Benavente, 2013).

\section{Análisis de los datos}

Los datos fueron sometidos a análisis descriptivos e inferenciales. Se comprobó, además, la normalidad de los mismos (Kolmogorov-Smirnov) y el índice de la consistencia interna de las diferentes escalas (Alfa de Cronbach). Para analizar las correlaciones entre las medidas objeto de estudio se utilizó el coeficiente bivariado de Pearson. La capacidad predictiva del autoconcepto sobre el perfil psicológico del deportista se evaluó mediante análisis de regresión lineal, utilizando el procedimiento de pasos sucesivos (Ruiz-Barquin, 2008). Para el procesamiento estadístico de los datos se ha usado el programa informatizado SPSS en su versión 20.0.

\section{Resultados}

La tabla 1 muestra los estadísticos descriptivos de las variables objeto de estudio. Como se puede observar, los valores de asimetría, curtosis y Kolmogorov-Smirnov indicaron que existía una distribución normal de los datos. Los ítems también presentan índices de normalidad adecuados (a excepción del 33, 34 y 41 del IPED). 
Tabla 1. Estadísticos descriptivos.

\begin{tabular}{|c|c|c|c|c|c|c|}
\hline & Media & DT & Asimetría & Curtosis & $Z$ & $p$ \\
\hline \multicolumn{7}{|l|}{ AF5 } \\
\hline Académico/profesional & 8.51 & 0.89 & -0.79 & 0.83 & 0.72 & .67 \\
\hline Social & 6.02 & 0.64 & -0.45 & -0.12 & 0.63 & .82 \\
\hline Emocional & 6.42 & 1.88 & -0.08 & -0.86 & 0.64 & .81 \\
\hline Familiar & 6.39 & 0.61 & -0.79 & 0.44 & 1.25 & .09 \\
\hline Físico & 7.53 & 1.19 & -0.33 & -0.34 & 0.49 & .97 \\
\hline \multicolumn{7}{|l|}{ IPED } \\
\hline Autoconfianza & 23.20 & 3.80 & -0.16 & -0.65 & 0.75 & .63 \\
\hline Afrontamiento negativo & 22.44 & 4.19 & -0.48 & -0.10 & 1.24 & .09 \\
\hline Control atencional & 22.01 & 3.71 & -0.58 & 1.20 & 1.16 & .14 \\
\hline Control viso-imaginativo & 22.28 & 4.62 & -0.49 & -0.10 & 0.93 & .35 \\
\hline Nivel motivacional & 23.38 & 3.13 & -0.27 & -0.24 & 0.94 & .34 \\
\hline Afrontamiento positivo & 24.75 & 3.33 & -0.63 & 0.52 & 1.01 & .27 \\
\hline Control actitudinal & 24.18 & 3.55 & -0.69 & 0.04 & 1.28 & .08 \\
\hline
\end{tabular}

$Z=$ Kolmogorov - Smirnov

En la tabla 2 se pueden observar las correlaciones (Pearson) establecidas entre las variables.

Tabla 2. Nivel de correlación (Pearson).

\begin{tabular}{llllll}
\hline & \multicolumn{3}{c}{ AF5 } \\
\cline { 2 - 6 } & \multicolumn{1}{c}{ Académico/ Profesional } & Social & Emocional & Familiar & Físico \\
\hline IPED & $.32^{* *}$ & $.22^{*}$ & $.39^{* * *}$ & $.22^{*}$ & $.29^{* *}$ \\
Autoconfianza & .11 & .06 & $.49^{* * *}$ & $.35^{* * *}$ & .19 \\
Afrontamiento negativo & .10 & .05 & $.26^{*}$ & $.25^{*}$ & .19 \\
Control atencional & .15 & .03 & .17 & -.18 & .14 \\
Control viso-imaginativo & $.26^{*}$ & .19 & .15 & .01 & .12 \\
Nivel motivacional & $.25^{*}$ & .18 & $.35^{* * *}$ & $.23^{*}$ & .17 \\
Afrontamiento positivo & $.28^{* *}$ & $.28^{* *}$ & $.43^{* * *}$ & $.25^{*}$ & .18 \\
Control actitudinal & & &
\end{tabular}

${ }^{*} p<.05 ;{ }^{* *} p<.01 ;{ }^{* * *} p<.001$

En la tabla 3 se muestran los análisis de regresión lineal efectuados (utilizando la técnica de pasos sucesivos), siendo las variables predictoras en cada modelo las dimensiones del autoconcepto y las variables criterio cada factor del Inventario Psicológico de Ejecución Deportiva. Este procedimiento ha sido utilizado para identificar aquellos factores del autoconcepto que permiten pronosticar el valor de las variables del perfil psicológico deportivo, indicando la variable con mayor capacidad de predicción y el modelo más adecuando. Las variables excluidas en los diversos casos no están presentes por falta de significación $(p>.05)$. Asimismo, no se muestra modelo para control viso-imaginativo por falta de significación. Los resultantes cumplen los supuestos de aceptación del modelo, como la linealidad en la relación entre variables predictoras y criterio, así como la homocedasticidad y distribución normal de los residuos, cuyo valor medio es 0 y la desviación típica prácticamente 1 (.99). Además, los valores de Durbin-Watson son adecuados, dado que se encuentran en un rango entre 1.77 y 2.18. Pardo y Ruiz (2005) consideran que cuando el estadístico se encuentra entre 1.5 y 2.5 se puede asumir que los residuos son independientes, cumpliéndose el supuesto de independencia de las variables independientes con respecto a la dependiente. Por otro lado, los estadísticos de colinealidad indican valores adecuados de inflación de la varianza (entre 1.00 y 1.01) y del índice de Tolerancia (entre .99 y 1.00) (Hair, Anderson, Tatham y Black, 1999). 
Tabla 3. Análisis de regresión lineal (pasos sucesivos)

\begin{tabular}{|c|c|c|c|c|c|c|c|c|c|}
\hline Variable criterio (IPED) & Modelo & $\mathrm{R}$ & $\mathrm{R}^{2}$ corregida & D-W & Variables Predictoras (AF5) & Beta & $\mathrm{T}$ & $\mathrm{T}$ & FIV \\
\hline \multirow[t]{9}{*}{ Autoconfianza } & 1 & .39 & .15 & & (Constante) & & $13.49^{* * *}$ & & \\
\hline & & & & & Emocional & .393 & $3.97^{* * *}$ & 1.00 & 1.00 \\
\hline & 2 & .50 & .23 & & (Constante) & & $2.04^{*}$ & & \\
\hline & & & & & Emocional & .372 & $3.99^{* * *}$ & .99 & 1.01 \\
\hline & & & & & Académico & .305 & $3.21^{* *}$ & .99 & 1.01 \\
\hline & 3 & .53 & .26 & 2.11 & (Constante) & & 0.06 & & \\
\hline & & & & & Emocional & .362 & $3.89^{* * *}$ & .99 & 1.01 \\
\hline & & & & & Académico & .300 & $3.23^{* *}$ & .99 & 1.01 \\
\hline & & & & & Físico & .186 & $1.99^{*}$ & .99 & 1.01 \\
\hline \multirow[t]{5}{*}{ Afrontamiento (-) } & 1 & .49 & .23 & & (Constante) & & $11.01^{* * *}$ & & \\
\hline & & & & & Emocional & .485 & $5.15^{* * *}$ & 1.00 & 1.00 \\
\hline & 2 & .58 & .32 & 2.18 & (Constante) & & 0.57 & & \\
\hline & & & & & Emocional & .461 & $5.17^{* * *}$ & .99 & 1.01 \\
\hline & & & & & Familiar & .309 & $3.47^{* *}$ & .99 & 1.01 \\
\hline \multirow[t]{5}{*}{ Control atencional } & 1 & .26 & .07 & & (Constante) & & $13.60^{* * *}$ & & \\
\hline & & & & & Emocional & .257 & $2.46^{*}$ & 1.00 & 1.00 \\
\hline & 2 & .35 & .10 & 1.77 & (Constante) & & $2.45^{*}$ & & \\
\hline & & & & & Emocional & .238 & $2.33^{*}$ & .99 & 1.01 \\
\hline & & & & & Familiar & .232 & $2.76^{*}$ & .99 & 1.01 \\
\hline \multirow[t]{2}{*}{ Nivel motivacional } & 1 & .26 & .06 & 1.94 & (Constante) & & $4.95^{* * *}$ & & \\
\hline & & & & & Académico & .264 & $2.54^{*}$ & 1.00 & 1.00 \\
\hline \multirow[t]{9}{*}{ Afrontamiento (+) } & 1 & .35 & .11 & & (Constante) & & $17.30^{* * *}$ & & \\
\hline & & & & & Emocional & .350 & $3.47^{* * *}$ & 1.00 & 1.00 \\
\hline & 2 & .42 & .16 & & (Constante) & & $4.07^{* * *}$ & & \\
\hline & & & & & Emocional & .337 & $3.42^{* * *}$ & .99 & 1.01 \\
\hline & & & & & Académico & .237 & $2.40^{*}$ & .99 & 1.01 \\
\hline & 3 & .47 & .19 & 2.12 & (Constante) & & 1.48 & & \\
\hline & & & & & Emocional & .321 & $3.32^{* *}$ & .99 & 1.01 \\
\hline & & & & & Académico & .233 & $2.42^{*}$ & .99 & 1.01 \\
\hline & & & & & Familiar & .199 & $2.05^{*}$ & .99 & 1.01 \\
\hline \multirow[t]{9}{*}{ Control actitudinal } & 1 & .43 & .17 & & (Constante) & & $15.39^{* * *}$ & & \\
\hline & & & & & Emocional & .427 & $4.37^{* * *}$ & 1.00 & 1.00 \\
\hline & 2 & .50 & .23 & & (Constante) & & $3.08^{* *}$ & & \\
\hline & & & & & Emocional & .412 & $4.38^{* * *}$ & .99 & 1.01 \\
\hline & & & & & Académico & .261 & $2.77^{* *}$ & .99 & 1.01 \\
\hline & 3 & .54 & .27 & 2.11 & (Constante) & & 0.61 & & \\
\hline & & & & & Emocional & .396 & $4.30^{* * *}$ & .99 & 1.01 \\
\hline & & & & & Académico & .258 & $2.81^{* *}$ & .99 & 1.01 \\
\hline & & & & & Familiar & .211 & $2.30^{*}$ & .99 & 1.01 \\
\hline
\end{tabular}

${ }^{*} p<.05 ;{ }^{* *} p<.01 ;{ }^{* * *} p<.001$ 


\section{Discusión}

El objetivo del presente estudio era analizar las relaciones entre el autoconcepto multidimensional y el perfil psicológico en triatletas, así como la capacidad predictiva del autoconcepto sobre las diferentes habilidades psicológicas contempladas en el Inventario Psicológico de Ejecución Deportiva (IPED). Los resultados han mostrado asociaciones significativas entre los constructos estudiados y el autoconcepto ha sido un buen predictor de la autoconfianza, el afrontamiento negativo y positivo, así como del control actitudinal.

En general, los análisis de correlación indican una relación positiva entre las variables analizadas, confirmando lo señalado por otros autores que habían puesto de relieve estas asociaciones en diferentes contextos (Diehl y Hay, 2010; Sung, 2011; Zhou et al., 2012). En el ámbito deportivo Jackson et al. (2001) habían seńalado que existían relaciones entre los juicios de autoconcepto, las habilidades psicológicas y el estado de flow, lo que se sitúa en la línea de las estimaciones de esta investigación y permite sugerir la importancia del autoconcepto en el desarrollo de las habilidades psicológicas del deportista. Sin embargo hay que destacar que el control viso-imaginativo no mostró relaciones con ninguna dimensión del autoconcepto y el nivel motivacional únicamente con el autoconcepto académico/profesional, lo cual no cumple las expectativas a la luz de la numerosa literatura que ha puesto de relieve los vínculos entre la motivación y el autoconcepto en diversos escenarios (Eyal y Roth, 2011; Faye y Sharpe, 2008; Martín-Albo et al., 2012; Wang et al., 2009).

Todas las dimensiones del autoconcepto han correlacionado con la autoconfianza, constituyendo uno de los modelos de regresión por pasos sucesivos los factores emocional, académico/profesional y físico. Diferentes investigadores han puesto de relieve las implicaciones de las percepciones de competencia y habilidad en la confianza de los deportistas, siendo además determinantes de la motivación desarrollada y el éxito deportivo (Aróstegi, Gońi, Zubillaga e Infante, 2013; Hagger, Hein y Chatzisarantis, 2011; Heazlewood y Burke, 2011; Montero, Moreno, González y Cervelló, 2013; Wang et al., 2009). En esta línea, Jaenes, Peñaloza, Navarreta y Bohórquez (2012) señalaron las relaciones negativas entre la autoconfianza y la ansiedad competitiva en triatlón, lo que pone de relieve el interés por analizar esta variable para evaluar la probabilidad de un adecuado rendimiento. En este deporte existe carencia de estudios que profundicen en la relación entre el autoconcepto y los perfiles psicológicos, aunque en un trabajo efectuado por Heazlewood y Burke (2011) sugieren que aspectos relacionados con la percepción de capacidad y habilidad podrían condicionar la confianza del atleta para afrontar la competición.

La capacidad de afrontamiento, tanto positivo como negativo, han sido también dos habilidades cuyos valores han podido ser predichos a partir del autoconcepto. Además, coinciden en que las dimensiones emocional y familiar han tenido una relación significativa con ellas. En particular, el control de afrontamiento negativo es la habilidad que mejor valor predictivo muestra respecto al resto de habilidades del perfil psicológico, siendo la asociación entre esta variable y el autoconcepto emocional la más elevada en este trabajo. En población general, ha quedado bien descrito en la literatura que el autoconcepto es una variable que está relacionada con la capacidad de afrontamiento (Radel, Pelletier y Sarrazin, 2013; Sung, 2011; Zhou et al., 2012). En el contexto deportivo es difícil encontrar trabajos que analicen la relación entre el autoconcepto multidimensional y la capacidad de afrontamiento, siendo su estudio en triatletas un ámbito inexplorado.

Por último, se puede destacar el modelo predictivo generado sobre el control actitudinal, en el que el autoconcepto emocional es el factor que obtiene un mayor peso. Esto tiene coherencia con el modelo multidimensional presentado en el IPED, dado que esta capacidad hace referencia al control sobre el pensamiento y la predisposición para la acción, y está relacionada con aspectos como el control emocional o la concentración (Hernández-Mendo, 2006; López-Gullón, 2011). Un buen autoconcepto podría influir en los pensamientos y actitudes vinculados a la competición, siendo más positivos y manifestando una mayor sensación de control (Álvarez, Falco, Estevan, Molina-García y Castillo, 2013). Específicamente, poseer una adecuada percepción del concepto emocional sugiere considerar al deportista como una persona que es consciente de sus emociones, es capaz de regularlas y evitar que puedan generar conductas desadaptativas en el periodo competitivo.

Entre las limitaciones del presente trabajo se encuentra el tamaño de la muestra. Aunque el acceso a la este tipo de población tiene una elevada dificultad se debería ampliar el número de participantes en posteriores trabajos para incrementar la validez externa del estudio. Además, el rango de edad de los triatletas era sensiblemente amplio lo que podría distorsionar los resultados. Debido a ello, sería interesante abordar estas cuestiones analizando en función de franjas de edad más ajustadas, para observar si se reproducen los resultados encontrados. Asimismo, se podría controlar también el nivel de rendimiento del triatleta para observar si esta variable genera diferencias en los resultados obtenidos. En futuras investigaciones será interesante, además, analizar las relaciones causa-efecto, dado que se podrá aportar así una visión más ajustada de los vínculos existentes entre estas variables.

A pesar de estas limitaciones, esta investigación supone profundizar en aspectos que no han sido estudiados en profundidad en triatletas y aporta información sobre la relación del autoconcepto con el perfil psicológico. Aunque se necesita una mayor evidencia sobre estos aspectos, los resultados encontrados sugieren la existencia de relaciones entre los juicios 
personales efectuados por los triatletas y la configuración de las habilidades psicológicas vinculadas al rendimiento deportivo. Por ello, este tipo de análisis podría ayudar a los profesionales que trabajan con estos atletas a detectar y mejorar circunstancias que pudieran afectar al éxito en competición., prestando atención al balance existente entre estas variables y observando la incidencia que podría tener en el rendimiento deportivo.

\section{Referencias}

1. Allen, M.S., Frings, D. y Hunter, S. (2012). Personality, coping, and challenge and threat states in athletes. International Journal of Sport and Exercise Psychology, 10(4), 264-275.

2. Álvarez, O., Falco, C., Estevan, I., Molina-García, J. y Castillo, I. (2013). Intervención psicológica en un equipo de gimnasia rítmica deportiva: Estudio de un caso. Revista de Psicología del Deporte, 22(2), 395-401.

3. Amesberger, G., Finkenzeller, T., Würth, S. y Müller, E. (2011). Physical self-concept and physical fitness in elderly individuals. Scandinavian Journal of Medicine \& Science in Sports, 21(s1), 83-90.

4. Aróstegi, B., Gońi, A., Zubillaga, A. y Infante, G. (2013). El autoconcepto físico de jóvenes futbolistas de alto rendimiento. Cuadernos de Psicología del Deporte, 13(1), 9-14.

5. Arruza, J.A., González, O., Palacios, M., Arribas, S. y Cecchini, J.A. (2012). Validación del Competitive State Anxiety Inventory 2 Reducido (CSAI-2 RE) mediante una aplicación web. Revista Internacional de Medicina y Ciencias de la Actividad Física y el Deporte, 12(47), 539-556.

6. Ato, M., López, J.J. y Benavente, A. (2013). Un sistema de clasificación de los diseńos de investigación en psicología. Anales de Psicología, 29(3), 1038-1059.

7. Berengüí, R., García-Pallarés, J., López-Gullón, J.M., Garcés de Los Fayos, E.J., Cuevas-Caravaca, E. y Martínez-Abellán, A. (2012). Habilidades psicológicas fundamentales en las Luchas Olímpicas. Cuadernos de Psicología del Deporte, 12(2), 19-22.

8. Cejuela, D.R., Pérez, D., Villa, D., Cortell, D. y Rodríguez, D. (2007). Análisis de los factores de rendimiento en triatlón distancia sprint. Journal of Human Sport \& Exercise, 2(2), 1-25.

9. Diehl, M. y Hay, E.L. (2010). Risk and resilience factors in coping with daily stress in adulthood: the role of age, self-concept incoherence, and personal control. Developmental Psychology, 46(5), 1132-1146.

10. Esnaola, I., Goñi, A. y Madariaga, J.M. (2008). El autoconcepto: perspectivas de investigación. Revista de Psicodidáctica, 13(1), 179-194.

11. Esnaola, I., Rodríguez, A. y Goñi, E. (2011). Propiedades psicométricas del cuestionario de Autoconcepto AF5. Anales de Psicología, 27(1), 109-117.

12. Eyal, O. y Roth, G. (2011). Principals' leadership and teachers' motivation: Self-determination theory analysis. Journal of Educational Administration, 49(3), 256-275.

13. Faye, C. y Sharpe, D. (2008). Academic motivation in university: The role of basic psychological needs and identity formation. Canadian Journal of Behavioural Science, 40(4), 189-199.

14. Feltz, D.L. (2007). Self-confidence and sports performance. En D. Smith y M. Bar-Eli (Eds.), Essential readings in sport and exercise psychology. Champaign: Human Kinetics.

15. Fernandes, H.M., Bombas, C., Lázaro, J.P. y Vasconcelos-Raposo, J. (2007). Perfil psicológico e sua importância no rendimento em vela. Motricidade, 3(3), 24-32.

16. García, F. y Musitu, G. (2001). Autoconcepto Forma 5. AF5. Manual. Madrid: TEA.

17. Garcia-Mas, A., Palou, P., E Smith, R., Ponseti, X., Almeida, P., Lameiras, J., Jiménez, R. y Leiva, A. (2011). Ansiedad competitiva y clima motivacional en jóvenes futbolistas de competición, en relación con las habilidades y el rendimiento percibido por sus entrenadores. Revista de Psicología del Deporte, 20(1), 197-207.
18. González-Ruiz, S.L., Hernández-Mendo, A., Pastrana Brincones, J.L. (2010). Herramienta software para la evaluación psicosocial de deportistas y entornos deportivos. Lecturas: EF y Deportes. Revista Digital, 15(144), mayo. [Consulta: 29 de mayo de 2010].

19. Gucciardi, D.F. (2012). Measuring mental toughness in sport: a psychometric examination of the Psychological Performance Inventory-A and its predecessor. Journal of Personality Assessment, 94(4), 393-403.

20. Guillén, F. y Ramírez, M. (2011). Relación entre el autoconcepto y la condición física en alumnos del Tercer Ciclo de Primaria. Revista de Psicología del Deporte, 20(1), 45-59.

21. Hagger, M.S., Hein, V. y Chatzisarantis, N.L.D. (2011). Achievement goals, physical self-concept, and social physique anxiety in a physical activity context. Journal of Applied Social Psychology, 41(6), 1299-1339.

22. Hair, J.F., Anderson, R.E., Tatham, R.L. y Black, W. (1999). Análisis multivariante ( $5^{\mathrm{a}} \mathrm{ed}$.). Madrid: Prentice Hall.

23. Hays, K., Thomas, O., Maynard, I. y Bawden, M. (2009). The role of confidence in world-class sport performance. Journal of Sports Sciences, 27(11), 1185-1199.

24. Heazlewood, I. y Burke, S. (2011). Self-efficacy and its relationship to selected sport psychological constructs in the prediction of performance in ironman triathlon. Journal of Human Sport \& Exercise, 6(2), 328-350.

25. Hernández-Mendo, A. (2006). Cuestionario para la evaluación psicológica de la ejecución deportiva: estudio complementario entre TCT y TRI. Revista de Psicología del Deporte. 15(1), 71-93.

26. Hernández-Mendo, A., Morales-Sánchez, V. y Peñalver, I. (2014). Replicación de las propiedades psicométricas del inventario psicológico de ejecución deportiva. Revista de Psicología del Deporte, 23(2), 311-324.

27. Jackson, S.A., Thomas, P.R., Marsh, H.W. y Smethurst, C.J. (2001). Relationships between flow, self-concept, psychological skills, and performance. Journal of Applied Sport Psychology, 13(2), 129-153.

28. Jaenes, J.C., Peńaloza, R., Navarrete, K.G. y Bohórquez, Ma.R. (2012). Ansiedad y autoconfianza precompetitiva en triatletas. Revista Iberoamericana de Psicología del Ejercicio y el Deporte, 7(1), 113-124.

29. Laborde, S., Dosseville, F. y Kinrade, N. (2014). Decision-specific reinvestment scale: an exploration of its construct validity, and association with stress and coping appraisals. Psychology of Sport and Exercise, 15(3), 238-246.

30. Landers, G.J., Ong, K.B., Ackland, T.R., Blanksby, B.A., Main, L.C. y Smith, D. (2013). Kinanthropometric differences between 1997 World championship junior elite and 2011 national junior elite triathletes. Journal of Science and Medicine in Sport, 16(5), 444-449.

31. Lepers, R., Knechtle, B. y Stapley, P.J. (2013). Trends in triathlon performance: Effects of sex and age. Sports Medicine, 43(9), 851-863.

32. Loehr, J. E. (1986). Mental Toughness Training for Sports: Achieving Athletic Excellence. Lexington: Stephen Greene Press.

33. Loehr, J.E. (1990). The Mental Game. New York: Plum Book.

34. López-Gullón, J.M., García-Pallarés, J., Berengüi, R., Martínez-Moreno, A., Morales-Baños, V., Torres-Bonete, M.D. y Díaz, A. (2011). Factores físicos y psicológicos predictores del éxito en lucha olímpica. Revista de Psicologia del Deporte, 20(2), 573-588.

35. Lopez-Gullon, J.M., Torres, M.D., Berengui, R., Diaz, A., Martinez, A., Morales, V. y Garcia-Pallares, J. (2012). Rendimiento fisico y psicológico en lucha olimpica: Predictores del éxito en lucha femenina. Anales de Psicología, 28(1), 215-222. 
36. Luszczynska, A. y Abraham, C. (2012). Reciprocal relationships between three aspects of physical self-concept, vigorous physical activity, and lung function: A longitudinal study among late adolescents. Psychology of Sport and Exercise, 13(5), 640-648.

37. Martín-Albo, J., Nún̄ez, J.L., Domínguez, E., León, J. y Tomás, J.M. (2012). Relationships between intrinsic motivation, physical self-concept and satisfaction with life: A longitudinal study. Journal of Sports Sciences, 30(4), 337-347.

38. McDonough, M.H. y Crocker, P.R. (2005). Sport participation motivation in young adolescent girls: The role of friendship quality and self-concept. Research Quarterly for Exercise and Sport, 76(4), 456-467.

39. Marongiu, E., Crisafulli, A., Pinna, M., Ghiani, G., Degortes, N., Concu, A. y Tocco, F. (2013). Evaluation of reliability of field tests to predict performance during Ironman Triathlon. Sport Sciences for Health, 9(2), 65-71.

40. Massuça, L.M., Fragoso, I. y Teles, J. (2014). Attributes of top elite team-handball players. The Journal of Strength \& Conditioning Research, 28(1), 178-186.

41. Montero, C., Moreno, J.A., González, D. y Cervelló, E.M. (2013). Motivación, dirección de la autoconfianza y flow en judokas de alto nivel. Motricidad: European Journal of Human Movement, 31, 1-16.

42. Nicholls, A.R. y Polman, R.C. (2007). Coping in sport: A systematic review. Journal of Sports Sciences, 25(1), 11-31.

43. Pardo, A. y Ruiz, M.A. (2005). Análisis de datos con SPSS 13 Base. Madrid: McGraw Hill.

44. Parry, D., Chinnasamy, C., Papadopoulou, E., Noakes, T. y Micklewright, D. (2011). Cognition and performance: anxiety, mood and perceived exertion among Ironman triathletes. British Journal of Sports Medicine, 45(14), 1088-1094.
45. Radel, R., Pelletier, L. y Sarrazin, P. (2013). Restoration processes after need thwarting: When autonomy depends on competence. Motivation and Emotion, 37(2), 234-244.

46. Ruiz-Barquín, R. (2008). Aportaciones del análisis subdimensional del cuestionario de personalidad BFQ para la predicción del rendimiento en judokas jóvenes de competición. Cuadernos de Psicología del Deporte, $8(1), 5-29$.

47. Shavelson, R.J., Hubner, J.J. y Stanton, J.C. (1976). Self-concept: validation of construct interpretations. Review of Educational Research, 46(3), 407-441.

48. Slutzky, C.B. y Simpkins, S.D. (2009). The link between children's sport participation and selfesteem: exploring the mediating role of sport self-concept. Psychology of Sport and Exercise, 10(3), 381-389

49. Sung, K.M. (2011). Self-concept and coping skills of female early adolescents in South Korea. The Journal of School Nursing, 27(5), 364-371.

50. Taylor, D. y Smith, M.F. (2013). Scalar-linear increases in perceived exertion are dissociated from residual physiological responses during sprint-distance triathlon. Physiology \& behavior, 118, 178-184.

51. Urra, B. (2014). Evaluación de la efectividad del entrenamiento de estrategias de afrontamiento en el nivel de ansiedad precompetitiva en tenimesistas. Revista de Psicología del Deporte, 23(1), 67-74.

52. Wang, J.C., Liu, W.C., Lochbaum, M.R. y Stevenson, S.J. (2009). Sport ability beliefs, $2 \times 2$ achievement goals, and intrinsic motivation: The moderating role of perceived competence in sport and exercise. Research Quarterly for Exercise and Sport, 80(2), 303-312.

53. Zhou, T., Wu, D. y Lin, L. (2012). On the intermediary function of coping styles: Between self-concept and subjective well being of adolescents of Han, Qiang and Yi nationalities. Psychology, 3(2), 136-142. 